\title{
Biogenerics and biobetters v/s chemical based drugs - comparative discourse
}

\begin{abstract}
In search of better drugs in terms of safety and efficacy of biological/biotherapeutics stand apart. The biotherapeutics being a technically innovative product require high intellectual inputs leading to capital expenditure. Biotherapeutics have definitely contributed in terms of efficacy, patient safety and quality of life for patients in terms of chemical based drugs. However, very high cost of manufacturing Biotherapeutics new innovations were initiated to create biogenerics mimicking the line of thought of generics of chemical origin. There are many biogenerics very launched in the market. However, the prices of biogenerics comparable high unlike prices of chemical based drugs. The biogenerics are not the same as the chemical based drugs because of inheritance difference in the structure and the brand innovator. Moreover some of the biogenerics had shown improved activity over the innovator brand are named as Biobetters.
\end{abstract}

Volume 5 Issue 3 - 2018

\author{
Anantha Naik Nagappa, Ramesh Basnet, \\ Asmit Acharya,Vinuth Chikkamath,Vaishnavi \\ Naik \\ Department of Pharmacy Practice, SCS College of Pharmacy, \\ India
}

Correspondence: Anantha Naik Nagappa, Department of Pharmacy Practice, SCS College of Pharmacy, Harapanahalli-583।3I, Karnataka, India, Email ananthal232000@gmail.com

Received: April 09, 2018 | Published: May 03, 2018

Keywords: biogenerics, biobetters, chromatography, human protein, biotherapeutics, hercpetine, biosimilars

Abbreviations: PCR, polymerization chain reaction; HPLC, high performance liquid chromatography; NMR, nuclear magnetic resonance; HIV, human immunodeficiency virus; WBC, white blood cells; RBC, red blood cells; DNA, deoxyribonucleic acid

\section{Introduction}

The biological products are in clinical practice since a century. For example, the insulin, growth hormone and antitoxins. After the decoding human genome in 2000, there were several biotechnology were developed which has led to revolutionaries in the field of clinical medicines never like before. The key technologies which made a major impact on biotechnology revolution recombinant DNA technology, hybridoma and Polymerization Chain Reaction (PCR) and many more. At the same time, there were advances in identification, isolation and characterization of new drugs by developing the instrumentations like
Nuclear Magnetic Resonance (NMR), Mass, High Performance Liquid Chromatography (HPLC) and Raman spectroscopy. Post genome in 2000 was a path breaking, as per the manufacturing process of biological prior to the 2000 the biological drugs which were obtained from natural sources like higher animals like sheep, horse, ox, etc. The products were extracted from the animal organs are like to cause hypersensitivity reactions in patients. The information technology with Biotechnology and instrumentation technology has given a shot in arm leading to availability of a variety of Biotherapeutics molecule in sufficient large number and quantity which can be used commercially for the treatment of disease. For example, human rDNA insulin and growth hormone is available in abundance for the treatment of the patients (Table 1). The Biotherapeutics molecules produced by biotechnological process are not having the drawbacks of the drugs obtained from the natural sources. ${ }^{1-3}$

Table I List of licensed Biotherapeutics with supporting documents ${ }^{4}$

\begin{tabular}{lll}
\hline Product & Trade name & Manufacturer \\
\hline Albumin & Albumin (Human) (None) & Octapharma Pharmazeutika Produktionsgesm.b.H \\
& Kedbumin & Kedrion Biopharmaceuticals, SpA \\
BCG Live & Tice BCG & Organon Teknika Corp \\
& BCG Vaccine & Organon Teknika Corp \\
Cholera Vaccine, Live, Oral & Vaxchora & Pax Vax Bermuda Ltd. \\
Diphtheria and tetanus toxoids & None [diphtheria and tetanus toxoids & Sanofi Pasteur, inc. \\
adsorbed, STN I03944 & adsorbed] & Roche diagnostics \\
Elecys HIV combi PT immunoassay & None [Elecys HIV combi PT immunoassay] & Octapharma Pharmazeutika Produktiondges.m.b.H. \\
Fibrinogen(human) & FIBRYNA & GlaxoSmithKline Biological \\
Hepatitis A vaccine, inactivated & Harvix & Protein sciences corporation \\
Influenza vaccine & Flublok & Wyeth pharmaceuticals, inc. \\
Meningococcal Group BVaccine & Trumenba & Merck \& Co,inc \\
\hline Pneumococcal vaccine, polyvalent & Pneumovax 23 &
\end{tabular}




\section{Biobetters}

While manufacturing the biogenerics it sometimes happens so that the biogenerics may show an improved efficacy and less toxicity than the innovator biotherapeutic brand in such circumsetences the biogeneric is considered as Biobetter or Biosuperior. However, it is highly technical field the biogenerics in making follows empirical apporoach and it is by chance and biobetter is produced. The molecular structural similarities are secondary to the biological activity and safety. Hence, more emphasis on efficacy and safety rather than the mimicking the structure of brand innovator. Under these circumsentances question arise is the credibility of patent laws concerning the Biotherapeutics. ${ }^{4,5}$ (Table 2).

Table 2 Overview: Comparison Biosimilars and Biobetters ${ }^{5}$

\begin{tabular}{|c|c|}
\hline Biosimilar & Biobetter \\
\hline $\begin{array}{l}\text { Biosimilars have limitations with respect } \\
\text { to structure i.e. they must be similar } \\
\text { active compound as that of reference } \\
\text { products. }\end{array}$ & $\begin{array}{l}\text { Biobetters do not have structural limitations, it may include molecular/chemical modifications, } \\
\text { and would therefore considered to have a different 'active compound' when compared to the } \\
\text { originator product. }\end{array}$ \\
\hline
\end{tabular}

Biosimilars should have somewhat similar safety and efficacy profiles

Biosimilars are very similar to innovator products.

Biobetters should have which has improved safety and/or efficacy profiles.

Biobetters are modified versions of innovator products.

Biosimilars are supposed to be approved after demonstrating similarity between biosimilar and reference product i.e. through comparability data.

Biobetters are like new drugs and supposed to travel through full new drug application or hybrid product application with all necessary clinical/non clinical trials data.

Biosimilars are not entitled to have patent protection or data exclusivity.

Biobetters may obtain patent or data exclusivity based on how innovative they are.

\section{Human protein}

The innovated biologist are soon patented and made available as a brand medicine with very high price. For example, the Hercpetine (Trastuzumab) a monoclonal antibody targeting breast cancer was made available at 24 lakhs (dollar) for one year treatment. The treatment with Hercpetine offered the advantages over conventional cytotoxic chemotherapy agents. The Herceptine is not having adverse drug reactions of conventional chemotherapeutic agents like alopecia, GIT destruction and bone marrow damage. This is possible the because the monoclonal antibody acted only on specific receptors present on the breast cancer cells. This drug would not interfere with other non breast cancer site. The monoclonal antibody efficacy is somewhat unpredictable and fear of allergic reaction has been always there for Biotherapeutics. The major drawback of MAB is sensitization of immune response.

The special advantage of MABS has created a high demand from the patients, Doctors, Pharmacists and Nurses due to its lack of hostile ADRs. Due to high demand and investment in developing the MAB had pushed the price of the all the MABs as frequent package for each MAB may run into several thousands of dollars this would have a negative impact on accessibility of the drugs of the MABs to the patients due to prohibiting policy. To address the high prices of MABs and other Biotherapeutics agents the stakeholders like Health Insurance provider, Governments, Drug regulators are requesting industry to keep the price of the medicine as low as possible because the issue of accessibility and affordability get addressed to a certain extent. Despite of the appeal Pharma corporate is arguing the prices cannot be reduced due to high developmental and promotional cost of the new medicines. The patent period of the biological is getting over and many Biologicals are available as biogeneric or biobetter of the innovator brand. The biogenric and Biobetters were cheaper compared to brand innovator. The governments and Health Insurance Company and regulators started encouraging the production of generic versions of Biotherapeutics.

Taking this opportunity several governments started encouraging the competitors of the innovator to take up the manufacture of generics of biological. Generics of biological should not be considered with generic drugs. The generic drugs of chemical origin can be quantified and correlated to its biological activity, for example ciprofloxacin etc. structural features of the biological drugs are important and determine the biological activity. The structural features of the biological drugs are likely to changes in environmental factors and also the conformational changes due to thermodynamic factors. Biological drugs are macromolecules unlike small chemical drugs. All these above factors lead to complexity and variability in the degree action. Hence, the biological drugs there can be batch variations and also the variations in Biosimilars and Biobetters is possible. The technology used by the innovator is a secrete proprietor matter which no corporation would like to share with the competitors with cell lines, processing and purifying technology has an infringing effect on the bioactivity of the biomarkers. Hence, it is not possible to develop the generic version of biological. In the making of Biosimilars some time the generic manufacturers' finds out a Biosimilars molecule which can perform better in terms of safety and efficacy than the innovator brand such Biosimilars are called Biobetters.

The existing cancer chemotherapy is neither safe nor cost effective. All conventional anticancer drug known to cause severe Adverse drug reactions which can lead to damage to gastric mucosa from mouth to anus. The drugs like Methotrexate, Hydroxy urea, Doxorubicin etc. are capable of suppressing the immune system and damaging 
the Haematopoietic tissue of bone marrow. They also cause damage to most of the actively dividing cells like Liver, Lungs and Gastric mucosa. The anticancer drugs are unable to discriminate healthy cells and cancerous cells. These effects lead to severe deficiency of blood cells like RBC, WBC and Platelets. As a result the quality of the life of the patients greatly disturbed by the alopecia, Nausea, Vomiting, etc. Further chemotherapeutic drugs are known to cause new cancers and severe suppression of the immune system leading to viral, fungal and bacterial infections in the patients. In order to find an alternative in the management of cancer Biotherapeutics were introduce. ${ }^{6-9}$ (Figure 1).

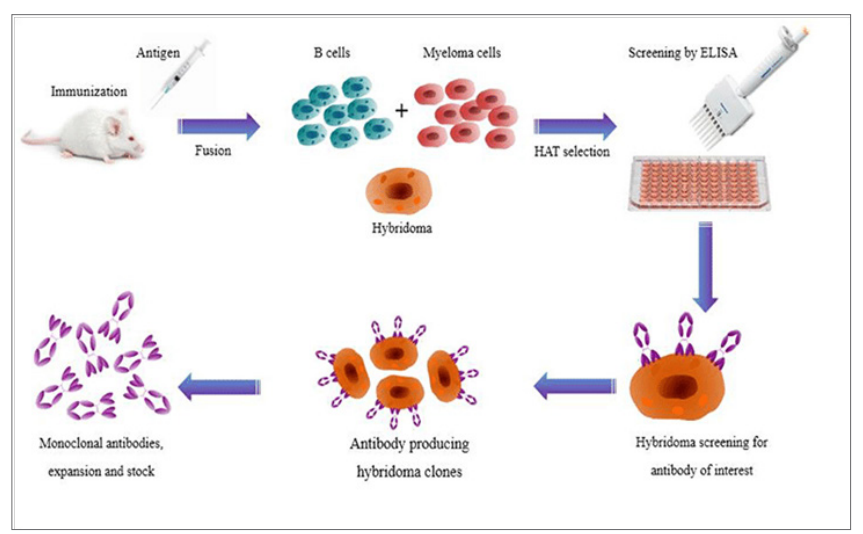

Figure I Shows the Biosimilars are developed from hybridroma technology. ${ }^{9}$

The utilization of recombinant technology along with hybdridoma technology has made it possible to synthesis any type of protein on an industrial scale. In this protocol the proteins from the cancer cells are identified and isolated in pure form. Thus purified proteins are injected to animal species to raise specific antibodies against the proteins of the cancer cells. These antibody genes is identified and isolated from the white blood cells. The genetic sequence of DNA is transferred to Murine stem cells. These stem cells can be cultured in the microbiological media shall start making the monoclonal antibodies which can be used as targeted drug delivery system against cancer which can only act cancer and sparing the other normal cells. Monoclonal antibodies are safe compared to chemotherapeutic agents which are devoid of toxicities of anticancer agents. These drugs are known to act, especially on cancer cells and sparing the normal cells. Targeting the cancer cells by MAB is a key factor for high specificity and least toxicity of the MAB. The only drawback with monoclonal antibodies is development of allergic reactions due to immune activation, unlike chemical therapeutic agents these drugs are macro molecules and are in fact proteins. The activity of these proteins is mainly dependent on structural integrity. The different manufactures who engage in manufacture of Biosimilars cannot guarantee the efficacy of a innovator brand Biotherapeutics agent. However, this is not the case with the conventional chemotherapeutic agents whose activity is predictable on chemical structure. Hence, the evaluation of chemical structure can utilize the various methods like IR, UV, and Visible spectroscopy along with mass spectroscopy, which principally measures the concentration of the drug in the biological fluids. Such an equation of dose response curve is not possible with Biosimilars. The Biosimilars are needed to be estimated in the activity units. Hence mandates the using of bioassays and is expressed in terms of activity units. ${ }^{10-13}$

\section{Conclusion}

Monoclonal Antibodies are able to target specific disease causing site and prevent the progression of the disease. However, as the therapy they lack a compressive cure for the treatment. The disease may be under control as long as treatment continued. As there is no guarantee that the disease may not come back again. MAB mostly never uses the single agent for radical cure they are used. As an adjuvant therapy it may help to reduce the toxicities of chemotherapy. Looking at the high cost, the economic burden prohibits liberal use of MABS in treatments.

Patent rights on Biotherapeutics are to be different than the IND small molecules, because there is a batch to batch variation in terms of structure and activity of the innovator product itself due to structural variations happens while manufacturing the biological activity evaluation cannot the consistent and predictable as in the case of IND small molecules. The biological activity should be the prime factor for patentability not its SAR.

Under such circumstances the Biosimilars manufacturer trains to imitate the technology of the innovator and attempts to make the biogenerics. In the process of manufacture, some of the generics manufacturers may be able to produce Biogenerics which are superior to innovator brand in terms of efficacy and safeties which are high lightened as Biobetters. Hence, the patenting the Biotherapeutics like patenting the IND is controversial. Regarding pricing of the Biotherapeutics, Biosimilars and Biobetters there is no control or rational leading to rule of Jungle makes them unaffordable for patients, health insurance provider, and also the governments' health schemes. The pricing policy should be critically evaluated so that the innovator should get a reasonable and return on investment and should not misuse. The patent loss for exploiting the customers on the basis of patent rights comparable to of small molecules IND. unless this challenge and issues are resolved by mutual understanding many of the biological and Biotherapeutics cannot be used just because the high cost of the medicines. It should be comparable to the issue of Anti HIV drugs which were priced ten thousand US dollars, which was brought down to six hundred US dollars by Cipla India, by manufacturing the AntiHIV drugs under the regimen of process patent as at the time India had recognized process and product patent as well.

\section{Acknowledgements}

The authors express deep sense of gratitude to the Management and Principal TMAE's SCS College of Pharmacy, Harapanahalli.

\section{Conflict of interest}

The author declares there is no conflict of interest.

\section{References}

1. Gabriel Bas T, Oliu Castillo C. Biosimilars in developed and developing East and Southeast Asian Countries Japan South Korea Malaysia overview Evolution and regulations assessment. BioMed Research International. 2016:12 p.

2. Jarrett S, Dingermann T. Biosimilars are here a hospital pharmacist's guide to educating health care professionals on biosimilars. Hosp Pharm. 2015;50(10):884-893. 
3. Nickisch K, Kerstin M Bode Greuel. Biosimilars or Biobetters make your decisions wisely. RAJAR. 2016;2(8):530-535.

4. Vaccines, Blood \& Biologics: Licensed Biological Products with Supporting Documents. US Food \& Drug Administration. 2018.

5. Sandeep V, Parveen J, Chauhan P. Biobetters the better biologics and their regulatory overview. IJDRA. 2016;4(1):13-20.

6. Eleryan MG, Akhiyat S, Rengifo Pardo M, et al. Biosimilars potential implications for clinicians. Clin Cosmet Investig Dermatol. 2016;9:135142.

7. Chapman K, Adjei A, Baldrick P, et al. Waiving in vivo studies for monoclonal antibody biosimilar development national and global challenges. MAbs. 2016;8(3):427-435.

8. Mysler E, Pineda C, Horiuchi T, et al. Clinical and regulatory perspectives on biosimilar therapies and intended copies of biologics in rheumatology. Rheumatol Int. 2016;36(5):613-625.
9. Illustration showing the production route of hybridoma technology Monoclonal antibodies. 2018.

10. Ebbers HC, Chamberlain P. Controversies in establishing biosimilarity extrapolation of indications and global labeling practices. Bio Drug. 2016;30(1):1-8

11. Mestre ferrandiz J, Towse A, Berdu M. Biosimilars how can payers get long term savings. PharmacoEconomics. 2016;34(6):609-616.

12. Jacobs I, Ewesuedo R, Lula S, et al. Biosimilars for the treatment of cancer a systematic review of published evidence. BioDrugs. 2017;31(1):1-36.

13. Liu Cheng, Morrow K. John Biosimilars of Monoclonal Antibodies: A Practical Guide to Manufacturing, Preclinical, and Clinical Development. Wiley \& Sons Ltd. 2017. 704 p. 